\title{
MEDICAMENTOS GENÉRICOS NOS ÚLTIMOS 20 ANOS E A PERCEPÇÃO DOS CONSUMIDORES
}

\section{GENERIC DRUGS IN THE LAST 20 YEARS AND CONSUMER PERCEPTION}

\author{
Larice de Amorim Rodrigues \\ Bacharel em Farmácia pela Faculdade de Almenara - ALFA. \\ E-mail: larice.amorim@yahoo.com.br \\ Thais de Oliveira Freitas \\ Bacharel em Farmácia pela Faculdade de Almenara - ALFA. \\ E-mail: thaiso.freitas22@hotmail.com
}

Viviane Amaral Toledo Coelho

Bióloga pelo Centro de Ensino Superior de Juiz de Fora; Especialista em Solos e Meio Ambiente pela Universidade Federal de Lavras; Mestre e Doutora em Ciência do Solo pela Universidade Federal de Lavras. Docente da Faculdade de Almenara - ALFA de Almenara - Minas Gerais. E-mail: vivianeatc@yahoo.com.br

\section{Carla Giselly de Souza}

Zootecnista pela Universidade Federal Rural de Pernambuco, Mestre em Produção Animal pela Universidade Júlio de Mesquita Filho-UNESP, Doutora em Nutrição de Ruminantes pela Universidade Federal da Paraíba, Pós-Doutoranda na Universidade Federal da Grande Dourados-MS. E-mail: carlaxlsouza@yahoo.com.br

\section{Luanna Botelho Souto de Araújo}

Farmacêutica/Bioquímica pela Universidade Presidente Antônio Carlos; Especialista em Análises Clinicas e toxicólogas pela Universidade Federal de Minas Gerais; Docente da Faculdade de Almenara - ALFA de Almenara - Minas Gerais. 


\section{RESUMO}

Avaliar na literatura científica as percepções dos consumidores com relação aos medicamentos genéricos no Brasil. Pesquisa bibliográfica com embasamento teórico sobre a percepção dos consumidores durante 20 anos do genérico. Apontaram que a variante do preço, a percepção dos consumidores ao longo dos anos foi majoritariamente que consideram menor o valor do medicamento genérico em relação ao medicamento de referência. No que tange a qualidade e segurança, o que se pode considerar também como confiança, é variável o que os pacientes consideram, onde acreditam que o medicamento genérico produz o mesmo efeito que 0 medicamento de referência. A utilização e preferência dos medicamentos genéricos e os fatores estão ligados ao preço e percebe-se que a população utiliza sim o medicamento genérico, mas ainda não é sua maior preferência. A percepção obtida com base na literatura cientifica apontou que os majoritariamente que no decorrer dos 20 anos, os consumidores consideram o medicamento genérico com valor menor que o de referência. A qualidade e eficácia do medicamento genérico segundo os consumidores é considerável como igual o medicamento de referência. Quanto a utilização e preferência do medicamento genérico ainda é relativamente baixa mesmo em detrimento do valor ser menor que o de referência.

Palavras-chaves: Medicamento genérico. Genérico e consumidor. Genérico no Brasil.

\section{ABSTRACT}

To evaluate in the scientific literature consumer perceptions regarding generic drugs in Brazil. Bibliographic research with theoretical basis on the perception of consumers during 20 years of the generic. They pointed out that the price variance, the perception of consumers over the years was mostly that they consider the value of the generic drug lower than the reference drug. Regarding quality and safety, which can also be considered as trustworthy, what patients consider varies, where they believe that the generic medicine has the same effect as the reference medicine. The use and preference of generic drugs and factors are linked to price and it is clear that the population does use the generic drug, but it is not yet their greatest preference. The perception obtained based on the scientific literature pointed out that the majority that over the 20 years, consumers consider the generic drug with lower value than the reference. The quality and efficacy of the generic medicine according to consumers is considerable as equal to the reference medicine. The use and preference of the generic drug is still relatively low even to the detriment of the value being lower than the reference value.

Keywords: Generic drug. Generic and consumer. Generic in Brazil.

\section{INTRODUÇÃO}


No Brasil atualmente a grande maioria dos medicamentos estão distribuídos em três grupos denominados de referência, também conhecidos como: éticos, genéricos e similares. O medicamento de referência é o produto inovador registrado na Anvisa cuja eficácia, segurança e qualidade foram comprovadas cientificamente por ocasião do registro. Já a segunda classe, os genéricos são aqueles medicamentos similares a um produto de referência ou inovador, que se pretende ser com este intercambiável, geralmente produzido após a expiração ou renúncia da proteção patentária ou de outros direitos de exclusividade, comprovada a sua eficácia, segurança e qualidade. Os genéricos não possuem nome comercial, e são designados pela Denominação Comum Brasileira (DCB) (BRASIL, 1999; GUTTIER et al., 2017).

Já os medicamentos similares são aqueles que devem ser identificados por nome comercial ou marca e que contém o mesmo ou os mesmos princípios ativos, apresentam a mesma concentração, forma farmacêutica, via de administração, posologia e indicação terapêutica, preventiva ou diagnóstica, do medicamento de referência registrado na Anvisa. Podem diferir dos medicamentos de referência somente em características relativas ao tamanho e forma do produto, prazo de validade, embalagem, rotulagem, excipientes e veículos (BARATA-SILVA et al., 2017).

A utilização do tratamento medicamentoso é indispensável para a reabilitação e manutenção da saúde, sendo o seu uso imprescindível para que os pacientes tenham uma alternativa para a proteção e promoção da saúde. O cenário para o acesso aos medicamentos, não apenas no Brasil, mas no mundo, fez-se restrito durante muitas décadas, devido, principalmente, aos elevados custos atribuídos ao processo produtivo dos medicamentos, somado às inúmeras exigências para a regulamentação dos mesmos junto aos órgãos sanitários pelas indústrias farmacêuticas (BLATT et al., 2012).

A redução dos preços dos medicamentos genéricos é possível em função de menor investimento em marketing, nas despesas com desenvolvimento de princípios ativos e ensaios clínicos necessários em um produto inovador, a competição entre as indústrias farmacêuticas por maior mercado. Além do fato que, por lei, os 
medicamentos genéricos devem ser pelo menos $35 \%$ mais baratos quando comparados ao medicamento referência (BLATT et al., 2012).

A popularização do medicamento genérico no Brasil poderia ser ainda maior, o que contribuiria ainda mais para a redução dos gastos com medicação, e a ampliação do acesso aos mesmos pela população. Porém, existem fatores de resistência ao produto quanto a sua utilização como: a baixa disponibilidade nas farmácias, falta de estímulo na prescrição, falta de conhecimento por parte dos consumidores e médicos, bem com a ausência de orientação adequada para o uso (BARATA-SILVA et al., 2017). Segundo Guttier et al. (2017), a falta de prescrição com o nome genérico e a falta de conhecimento dos consumidores, estão entre as principais barreiras para uma maior popularização dos genéricos.

Completa-se no ano de 2019, 20 anos da implantação da política dos medicamentos genéricos no Brasil, e ainda existem alguns fatores que interferem positiva ou negativamente na popularização e na relação de confiança entre consumidores e os genéricos.

Desta forma, o presente trabalho tem como objetivo avaliar as percepções dos consumidores com relação aos medicamentos genéricos no Brasil. Bem como, esta percepção do consumidor ao longo dos 20 anos da implantação dos genéricos no Brasil, demonstrando os aspectos positivos e negativos em relação aos medicamentos genéricos no Brasil na perspectiva do consumidor.

\section{METODOLOGIA}

O presente trabalho, teve uma abordagem qualitativa partindo de uma pesquisa bibliográfica que buscou responder a seguinte questão: Quais são as percepções dos consumidores aos medicamentos genéricos no Brasil?

O embasamento teórico-científico englobou artigos científicos publicados entre os anos de 1999 a 2019 e relatórios oficiais dos órgãos públicos como Ministério da Saúde e Agência Nacional de Vigilância Sanitária (Anvisa). Os estudos indexados foram selecionados nas bases de dados: Biblioteca Virtual em Saúde Brasil (BVS), Literatura Latino-Americana e do Caribe em Ciências da Saúde 
(Lilacs), National Library of Medicine (Medline), Portal de Periódicos Capes e Scientific Electronic Library Online (Scielo).

Os descritores de busca nas bases de dados foram: "medicamento genérico", "genérico AND consumidor", "genérico no Brasil". Os critérios de seleção dos artigos partiram primeiramente com base na leitura do título e do resumo. Os artigos que foram selecionados passaram pelo último crivo de aprovação, realizando a leitura completa do artigo. Os artigos selecionados objeto do presente estudo apresentaram em seu conteúdo pareceres, percepções de consumidores brasileiros com relação aos medicamentos genéricos.

De posse da percepção dos consumidores relatadas nos artigos selecionados foram listados os principais pontos positivos e negativos da implantação da política de medicamentos genéricos no Brasil com base na visão dos consumidores. Todos esses pontos levantados foram discutidos com base na literatura científica e relatórios disponibilizados pelos órgãos oficiais.

\section{RESULTADOS E DISCUSSÃO}

Os medicamentos genéricos são considerados um marco na indústria farmacêutica brasileira, uma vez que são medicamentos com o mesmo princípio ativo dos medicamentos de referência com qualidade, segurança e eficiência asseguradas pela legislação (BERTOLDI et al., 2016).

A utilização dos medicamentos genéricos se popularizou e ganhou espaço no mercado, sendo uma solução viável, acessível e segura para a população. No ano de 2014 já se podia tratar a grande maioria das doenças predominantes no Brasil e a um custo de até $35,0 \%$ a $40 \%$ menor usando os genéricos em detrimento ao uso de medicamentos de referência (BLATT et al., 2012; BERTOLDI et al., 2016).

A implementação da política de medicamentos genéricos gerou um grande impacto para a indústria farmacêutica, uma vez que aumentou a concorrência entre os produtos comercializados e o genérico conquistou mercado. Houve um aumento de $90 \%$ das empresas produtoras de genéricos (SILVA; ROCHA, 2016; GUTTIER et al., 2017; BERTOLDI et al., 2016). A partir de então foi possível a regulação dos preços dos medicamentos no país por meio da Anvisa, atuando como secretaria 
executiva, pelo Ministério da Saúde e Câmara de Regulação do Mercado de Medicamentos - CMED (NISHIJIMA; BIASOTO; LAGROTERIA, 2014).

Alguns fatores influenciam na decisão de compra do medicamento pelos brasileiros estando entre eles a fidelidade à prescrição médica, o preço e a disponibilidade (SOUSA; MESQUITA; LARA, 2013). Pesquisas realizadas por Guttier et al. (2017) apontam que, a preferência por medicamentos genéricos é em torno de $60 \%$. O fator que predomina para a decisão de compra do medicamento é o poder aquisitivo. Outro fator determinante para a escolha do medicamento na ocasião da compra é a confiança. A relação de confiança existente tanto por parte dos prescritores, quanto pelos pacientes é fundamental para a substituição do medicamento referência pelo genérico (BLATT et al., 2012; SOUSA; MESQUITA; LARA, 2013; GUTTIER et al., 2016).

Em função do menor valor atribuído aos medicamentos genéricos, estudos de Bertoldi et al. (2016) na Pesquisa Nacional de Acesso, Utilização e Promoção do Uso Racional de Medicamentos (PNAUM), entre os anos de 2013 e 2014, apontaram que a prevalência do uso do genérico foi de $45,5 \%$, onde sua maior utilização foi na classe econômica C. Isso demonstra que a população necessita de maiores esclarecimentos com relação aos genéricos, uma vez que menos de $50 \%$ utilizam os genéricos, com ênfase de maior consumo pela classe de menor poder aquisitivo, provavelmente devido ao menor custo dos genéricos.

Uma pesquisa realizada no período de 2000 a 2004 por Carvalho e colaboradores (2015) apontaram que os medicamentos genéricos passaram a ser mais barato em relação ao medicamento de referência chegando a variar de 42,8\% no ano de 2000, chegando até 46,9\% em 2004.

Visando compreender a percepção dos consumidores em relação aos medicamentos genéricos ao longo da sua implantação no Brasil, compilou-se as pesquisas dos autores, traçando uma linha temporal com a variantes de preço, qualidade e utilização/preferência conforme Quadro 1:

Quadro 1 - Percepção dos consumidores ao longo dos anos em relação aos genéricos $(2002-2017)$.

\begin{tabular}{|l|c|c|c|c|c|c|}
\hline Autor & Local & Ano & Amostra / & Preço & Qualidade & Utilização/ \\
\hline
\end{tabular}




\begin{tabular}{|c|c|c|c|c|c|c|}
\hline & & & $\mathbf{N}$ & & & Preferência \\
\hline $\begin{array}{l}\text { Souza, } \\
\text { et al., } \\
2002\end{array}$ & $\begin{array}{l}\text { Londrina, } \\
\text { PR }\end{array}$ & 2002 & $\begin{array}{l}201 \\
\text { pacientes } \\
\text { com idade } \\
\text { acima de } 16 \\
\text { anos }\end{array}$ & - & $\begin{array}{l}76,7 \% \text { disseram } \\
\text { que o } \\
\text { medicamento } \\
\text { genérico tem o } \\
\text { mesmo resultado } \\
\text { do de marca }\end{array}$ & $\begin{array}{l}48,3 \% \text { compram } \\
\text { o medicamento } \\
\text { genérico }\end{array}$ \\
\hline $\begin{array}{l}\text { Bertoldi } \\
\text { et al., } \\
2005\end{array}$ & $\begin{array}{l}\text { Pelotas, } \\
\text { SC }\end{array}$ & 2002 & $\begin{array}{l}3182 \\
\text { adultos (20 } \\
\text { anos ou } \\
\text { mais) }\end{array}$ & $\begin{array}{l}86 \% \\
\text { disseram } \\
\text { que } \\
\text { genérico } \\
\text { mais barato }\end{array}$ & $\begin{array}{l}70 \% \text { acreditam } \\
\text { que a qualidade } \\
\text { dos genéricos } \\
\text { seja a mesma } \\
\text { que a dos } \\
\text { medicamentos } \\
\text { referência }\end{array}$ & - \\
\hline $\begin{array}{l}\text { Rocha } \\
\text { Barros } \\
\text { Silva, } \\
2007\end{array}$ & Recife, PE & 2003 & $\begin{array}{l}\text { Pacientes } \\
\text { setores de } \\
\text { saúde da } \\
\text { cidade de } \\
\text { Recife, } \\
\text { Idade maior } \\
\text { que } 21 \\
\text { anos. }\end{array}$ & $\begin{array}{l}68,9 \% \\
\text { afirmaram } \\
\text { ter o } \\
\text { medicament } \\
\text { o genérico } \\
\text { menor } \\
\text { preço que o } \\
\text { de marca } \\
\text { comercial } \\
\end{array}$ & - & - \\
\hline $\begin{array}{l}\text { Vosger } \\
\text { au, } \\
\text { Souza, } \\
\text { Soares, } \\
2011\end{array}$ & $\begin{array}{l}\text { Ponta } \\
\text { Grossa, } \\
\text { PR }\end{array}$ & $\begin{array}{l}2006 \text { e } \\
2007\end{array}$ & $\begin{array}{l}374 \text { adultos } \\
(20-59 \\
\text { anos) }\end{array}$ & $\begin{array}{l}88,9 \% \\
\text { disseram } \\
\text { que o valor } \\
\text { genérico é } \\
\text { menor que } \\
\text { o } \\
\text { medicament } \\
\text { o de } \\
\text { referência }\end{array}$ & $\begin{array}{l}64,3 \% \text { acreditam } \\
\text { que os genéricos } \\
\text { têm a mesma } \\
\text { qualidade } \\
\text { quando } \\
\text { comparados aos } \\
\text { medicamentos } \\
\text { de referência; }\end{array}$ & $\begin{array}{l}60,7 \% \text { tem } \\
\text { preferência pelo } \\
\text { medicamento } \\
\text { genérico. }\end{array}$ \\
\hline $\begin{array}{l}\text { Blatt et } \\
\text { al., } \\
2012\end{array}$ & $\begin{array}{l}\text { Tubarão, } \\
\text { SC }\end{array}$ & 2007 & $\begin{array}{l}234 \\
\text { pessoas } \\
\text { maiores de } \\
18 \text { anos }\end{array}$ & - & $\begin{array}{l}76,9 \% \text { acreditam } \\
\text { que os genéricos } \\
\text { têm o mesmo } \\
\text { efeito que os de } \\
\text { marca }\end{array}$ & $\begin{array}{l}\text { 85\% já utilizaram } \\
\text { medicamento } \\
\text { genérico }\end{array}$ \\
\hline $\begin{array}{l}\text { Fernan } \\
\text { des, } \\
\text { Coutinh } \\
\text { o e } \\
\text { Valle, } \\
2011\end{array}$ & $\begin{array}{l}\text { Brasília, } \\
\text { DF }\end{array}$ & 2009 & $\begin{array}{l}84 \\
\text { consumidor } \\
\text { es }\end{array}$ & - & - & $\begin{array}{l}85 \% \text { escolhem o } \\
\text { medicamento } \\
\text { genérico pelo } \\
\text { valor }\end{array}$ \\
\hline $\begin{array}{l}\text { Guttier } \\
\text { et al., } \\
2016 .\end{array}$ & $\begin{array}{l}\text { Rio } \\
\text { Grande do } \\
\text { Sul, Ponta } \\
\text { Grossa, } \\
\text { Paraná, } \\
\text { Tubarão, } \\
\text { Santa, } \\
\text { Catarina - } \\
\text { Região } \\
\text { SUL } \\
\end{array}$ & 2012 & $\begin{array}{l}3.372 \\
\text { pessoas }\end{array}$ & $\begin{array}{l}87 \% \\
\text { disseram } \\
\text { que o valor } \\
\text { genérico é } \\
\text { menor que } \\
\text { o } \\
\text { medicament } \\
\text { o de } \\
\text { referência }\end{array}$ & $\begin{array}{l}69,1 \% \text { dos } \\
\text { consumidores } \\
\text { apontaram } \\
\text { qualidade } \\
\text { equivalente entre } \\
\text { o medicamento } \\
\text { genérico e o } \\
\text { medicamento de } \\
\text { referencia }\end{array}$ & $\begin{array}{l}26,1 \% \text { utilizavam } \\
\text { os } \\
\text { medicamentos } \\
\text { genéricos }\end{array}$ \\
\hline $\begin{array}{l}\text { Sousa, } \\
\text { Mesqui }\end{array}$ & $\begin{array}{l}\text { Belo } \\
\text { Horizonte, }\end{array}$ & 2013 & $\begin{array}{l}403 \\
\text { consumidor }\end{array}$ & $\begin{array}{l}61,04 \% \\
\text { confirmam }\end{array}$ & - & $\begin{array}{l}85,9 \% \text { os } \\
\text { utilizaram o }\end{array}$ \\
\hline
\end{tabular}




\begin{tabular}{|c|c|c|c|c|c|c|}
\hline $\begin{array}{l}\text { ta e } \\
\text { Lara, } \\
2013 .\end{array}$ & MG & & es & $\begin{array}{l}\text { que os } \\
\text { preços } \\
\text { foram } \\
\text { reduzidos } \\
\text { em relação } \\
\text { ao de marca }\end{array}$ & & genérico \\
\hline $\begin{array}{l}\text { Lira et } \\
\text { al., } \\
2014\end{array}$ & $\begin{array}{l}\text { São } \\
\text { Paulo, } \\
\text { Goiás e } \\
\text { Rio de } \\
\text { Janeiro }\end{array}$ & 2014 & $\begin{array}{l}278 \\
\text { pacientes } \\
\text { com idade } \\
\text { de } \\
37,1 \pm 15,8 \\
\text { anos }\end{array}$ & $\begin{array}{l}88,8 \% \\
\text { informaram } \\
\text { que o } \\
\text { genérico } \\
\text { possuía um } \\
\text { preço } \\
\text { menor que } \\
\text { o } \\
\text { medicament } \\
\text { o de marca }\end{array}$ & $\begin{array}{l}74,8 \% \\
\text { acreditavam que } \\
\text { o medicamento } \\
\text { genérico possuía } \\
\text { um efeito igual } \\
\text { ao do } \\
\text { medicamento de } \\
\text { marca }\end{array}$ & - \\
\hline $\begin{array}{l}\text { Brito, } \\
2018\end{array}$ & $\begin{array}{l}\text { Lago } \\
\text { Verde, MA }\end{array}$ & 2017 & $\begin{array}{l}100 \\
\text { usuários }\end{array}$ & $\begin{array}{l}80 \%, \\
\text { considerara } \\
\text { m que o } \\
\text { baixo custo } \\
\text { do } \\
\text { medicament } \\
\text { o genérico } \\
\text { menor }\end{array}$ & $\begin{array}{l}60 \% \text { acredita que } \\
\text { possui o mesmo } \\
\text { efeito que o } \\
\text { medicamento de } \\
\text { referência }\end{array}$ & $\begin{array}{l}64 \% \text { faz o uso } \\
\text { para alguma } \\
\text { finalidade } \\
\text { terapêutica e }\end{array}$ \\
\hline $\begin{array}{l}\text { Alcânta } \\
\text { ra, } \\
2017\end{array}$ & $\begin{array}{l}\text { Juazeiro } \\
\text { do Norte, } \\
\text { CE }\end{array}$ & 2017 & $\begin{array}{l}69 \\
\text { farmácias } \\
\text { comerciais } \\
\text { na cidade }\end{array}$ & - & $\begin{array}{l}87 \% \text { acreditam } \\
\text { que o } \\
\text { medicamento } \\
\text { genérico produz } \\
\text { o mesmo efeito } \\
\text { que o de } \\
\text { referência }\end{array}$ & $\begin{array}{l}\text { 42\% Utilização } \\
\text { do medicamento } \\
\text { Genérico } \\
\text { motivado pelo } \\
\text { custo }\end{array}$ \\
\hline
\end{tabular}

Fonte: Elaborador pelos autores (2019).

Pode-se inferir no que diz respeito a variante do preço, a percepção dos consumidores ao longo dos anos foi majoritariamente que consideram menor o valor do medicamento genérico em relação ao medicamento de referência.

No que tange a qualidade e segurança, o que se pode considerar também como confiança, é a variável que os consumidores consideram, onde, acreditam que o medicamento genérico produz o mesmo efeito que o medicamento de referência. Foi possível perceber que a utilização e preferência pelos medicamentos genéricos estão ligados principalmente ao preço, e percebe-se que a população utiliza sim o medicamento genérico, mas ainda não é sua preferência majoritária.

Em síntese, logo após a implantação da Política de Medicamentos Genéricos do Brasil, no ano de 2002 os consumidores em sua grande maioria, consideravam o valor menor em relação ao medicamento de referência. Esta visão manteve-se nos anos subsequentes até o ano de 2017, conforme apresentado na Quadro 1. 
Concluindo-se que após 20 anos de implantação, a percepção dos consumidores é que os medicamentos genéricos possuem menor valor em detrimento ao medicamento de referência.

No que diz respeito a percepção dos consumidores em relação a qualidade, acreditam que o medicamento genérico possui o mesmo efeito e qualidade do medicamento de referência. No início esta percepção foi maior e com o passar dos anos teve um decréscimo. Mas de maneira geral, o quantitativo desta variante permanece em torno dos $70 \%$.

Com relação à percepção dos consumidores, conforme pode ser observado no Quadro 1, quanto a utilização e preferência, no ano de 2002, a preferência era pequena, onde poucos compravam o medicamento genérico, mesmo concordando com o preço menor. A partir do ano de 2006, esta preferência teve um aumento, apesar de ainda ser números pequenos.

Sendo assim, destaca-se a importância da atuação do profissional ao realizar uma prescrição ao paciente, pois muitas dessas barreiras são criadas por profissionais que ao realizar a prescrição deixam claro a não aceitação da troca do medicamento referência pelo genérico, aumentado assim as dúvidas da população quanto a eficácia e qualidade dos genéricos. Assim, podemos ver como papel da classe farmacêutica difundir orientações acerca do medicamento genérico no momento do atendimento ao cliente. Oferecendo o medicamento genérico, e explicando sua diferença e eficácia em relação a um medicamento referência, possibilitado assim uma melhor aceitação sobre os mesmos.

\section{CONSIDERAÇÕES FINAIS}

A Política dos medicamentos genéricos no Brasil, representa um grande marco na indústria farmacêutica e um ganho para a população, uma vez que possibilitou o acesso à medicamento com menor custo e com a mesma qualidade e eficácia, aumentado assim a chance de um melhor tratamento ao paciente.

A percepção obtida apontou que no decorrer dos 20 anos, os consumidores consideram o medicamento genérico com menor custo em relação ao de referência. 
Sendo esse fato garantido pela lei que os medicamentos genéricos devem ser pelo menos $35 \%$ a $40 \%$ mais baratos quando comparados ao medicamento referência.

Os consumidores consideraram que a qualidade e eficácia do medicamento genérico é igual ao medicamento de referência.

A utilização e preferência por medicamento genérico ainda é relativamente baixa. Deixando claro que no decorrer desses 20 anos de implantação dos medicamentos genéricos ainda existem barreiras sobre a sua aceitação.

\section{REFERÊNCIAS BIBLIOGRÁFICAS}

ALCÂNTARA, R. F. A percepção da população de consumidores de medicamentos genéricos em farmácias comerciais na região metropolitana do Cariri. BIOFARM, v. 13, n. 4, out. / dez. 2017.

BARATA-SILVA, C. et al. Desafios ao controle da qualidade de medicamentos no Brasil. Cad. Saúde Coletiva. Rio de Janeiro, v. 25, n. 3, p. 362-370, 2017.

BERTOLDI, A.D. et al. Utilização de medicamentos genéricos na população brasileira: uma avaliação da PNAUM 2014. Rev. Saúde Pública. v. 50, p. 1-11, 2016.

BLATT, C.R. et al. Conhecimento popular e utilização dos medicamentos genéricos na população do município de Tubarão, SC. Ciência \& Saúde Coletiva, v. 17, n. 1, p. 79-87, 2012.

BRASIL. Lei no 9.787, de 10 de fevereiro de 1999. Disponível em: <http://www.planalto.gov.br/ccivil_03/Leis/L9787.htm>. Acesso em 05 de abril 19.

\section{BRITO, J. A. Adesão ao uso de medicamentos genéricos em uma farmácia no} município de Lago Verde: MA. Farmacêutica da Faculdade Cathedral/Instituto Brasil de Pós-Graduação, Capacitação e Assessoria. São Luis, 2018. 
CARVALHO, B. et al. Influências culturais e sociais no uso de medicamentos genéricos por idosos no bairro avenida da cidade de Itajubá, Minas Gerais. Rev. Ciênc. Saúde, v. 5, n. 3, p. 15-21, 2015.

CONSELHO FEDERAL DE FARMÁCIA. Resolução n. 596 de 21 de fevereiro de 2014.

CONSELHO FEDERAL DE FARMÁCIA. Resolução no. 586 de $\mathbf{2 9}$ de agosto de 2013.

FERNANDES, J. A.; COUTINHO, J. V.; VALLE, M. G. Aceitação do medicamento genérico em diferentes níveis de Escolaridade e renda familiar do distrito federal.

Cenarium Pharmacêutico, ano 4, n. 4, mai. / nov. 2011.

GUTTIER, M. C. et al. Fatores que influenciam a preferência pela compra de medicamentos genéricos em uma cidade do sul do Brasil. Revista Saúde Pública, São Paulo, v. 51, 2017.

GUTTIER, M. C. et al. Percepção, conhecimento e uso de medicamentos genéricos no Sul do Brasil: o que mudou entre 2002 e 2012? Caderno Saúde Pública, Rio de Janeiro, v. 32, n. 7, p. 1-13, jul. 2016.

LIRA, C. A. B. et al. Conhecimento, percepções e utilização de medicamentos genéricos: um estudo transversal. Einstein, v. 12, n. 3, p. 267-73, 2014.

MONTEIRO, C. N. et al. Utilização de medicamentos genéricos no município de São Paulo em 2003: estudo de base populacional. Epidemiologia Serviço Saúde, Brasília, v. 25, n. 2, p. 251-

NISHIJIMA, M. A.; BIASOTO, G; LAGROTERIA, E. A competição no mercado farmacêutico brasileiro após uma década de medicamentos genéricos: uma análise 
de rivalidade em um mercado regulado. Economia e Sociedade, Campinas, v. 23, n. 1, p. 155-186, abr. 2014.

ROCHA, C. E.; BARROS, J. A. C.; SILVA, M. D. P. Levantamento de dados sobre o conhecimento e informação acerca dos medicamentos genéricos em uma população de pacientes do serviço de saúde ambulatorial do Recife, Pernambuco, Brasil. Caderno Saúde Pública, Rio de Janeiro, v. 23, n. 5, p. 1141-1150, mai. 2007.

SILVA, C. M. et al. Medicamentos genéricos: uma abordagem no município de Vale do Paraíso, Rondônia. Acta Biomedica Brasiliensia. v. 8, n. 2, p. 83-90, dez. 2017.

SALOMAO, P. E. A. et al. As Tecnologias de Informação e Comunicação (TIC) no ensino superior. Revista Multidisciplinar do Nordeste Mineiro, v. 1, 2018.

SILVA, N. C. S.; ROCHA, L. C. Medicamentos genéricos: legislação, política e mercado. Única Cadernos Acadêmicos, Ipatinga-MG, v. 3, n. 1, 2016.

SOUSA, C. V.; MESQUITA, J. M. C.; LARA, J. E. Análise da decisão de compra de medicamentos frente à existência de produtos substitutos: um estudo no município de Belo Horizonte, Brasil. Ciência \& Saúde Coletiva, v. 18, n. 11, p. 3311-3320, 2013.

SOUZA, M. J. B. et al. Análise da Percepção do Usuário de Medicamentos Genéricos: um enfoque de Marketing Social aplicado à saúde pública. Anais eletrônicos, Salvador, 2002.

VOSGERAU, M. Z. S.; SOUZA, R. K. T.; SOARES, D. A. Utilização de genéricos em área de atuação da equipe de Saúde da Família em município do sul do Brasil.

Revista Brasileira de Epidemiologia, v. 14, n. 2, p. 253-63, 2011. 
Revista Multidisciplinar do Nordeste Mineiro, v1. 2020/01 ISSN 2178-6925 